\title{
Gateway Load Balancing and Routing Selection Scheme of MANET in Disaster Scenario
}

\author{
Nor Aida Mahiddin ${ }^{1,2, *}$ Nurul I Sarkar ${ }^{1}$ and Brian Cusack ${ }^{1}$ \\ ${ }^{1}$ Auckland University of Technology (AUT), New Zealand \\ ${ }^{2}$ University Sultan Zainal Abidin (UniSZA), Malaysia \\ *Contact: nmahiddi@aut.ac.nz
}

\begin{abstract}
After disaster occurs like earthquake, it is common results of collapse structure. It is impossible for disaster victims make use of communication devices such as cellular phones, iPad or laptop to communicate with their family and friends who are trapped in the disaster when the devices become unworkable due to service communication failure in that disaster area. With infrastructure less and decentralize features, mobile ad hoc network (MANET) is ideal to be one of the options to solve this problem. Therefore, the important functionalities of MANET that allows users to create dynamically configurable wireless networks without fixed infrastructure, using common devices like mobile phones was very helpful. Nodes (victims or rescue team devices) can be a source, destination or relay of the information. The objective of this research is to develop a better gateway load balancing and routing selection scheme to improve performance of MANET in disaster scenario. This scheme designed to make the task even between gateways and to simplify the route selection. Results obtained have shown that the proposed schemes can reduce network congestion and consequently can achieve better network performances. Performances will be measured with several metrics such as fairness, throughput and packet delay.
\end{abstract}

Keywords: MANET, Gateway Load Balancing, Routing Selection and Disaster Scenario.

\section{INTRODUCTION}

Mobile Ad Hoc Network (MANET) is one type of Ad Hoc Network. Expansion of wireless technologies has brought data transmission via radio waves and nodes in the network communicated each other without fixed station access point. Therefore MANET is a self-configure and infrastructure less network. This form and deforms a network on the fly without relying on any network system. These decentralized characters give an advantage to the network. An advantage of decentralization is robustness, flexibility and mobility. MANET is gaining popularity since the production of smart computing device and development of wireless communication are increased. Transmission of information from source to destination across inter-network is called routing. To forward data packets from source to a destination, neighbour's node also known as routers (because perform data packet forwarding) will send the data packet through multihop node until the data packet arrive at the destination. Thus, topology network in MANET are known as unpredictable and can be change rapidly [1] IEEE 802.11 is a standard for wireless family. Two operational modes define by IEEE 802.11 standard: Infrastructure-based and Infrastructure less (Ad Hoc). Infrastructure based reacts as Wi-Fi hotspots that enabling devices connecting to Internet. However for dynamic environments, which people or devices that connected to Internet for temporary time, infrastructure less or ad hoc mode is more efficient. Nodes in this mode are performing Independent Basic Service Set (IBSS) or Ad Hoc Network. Every node can communicate each other after succeed in synchronization phase. If one of nodes (in this case we called it node A) in the network is connected to wire network, all nodes have wireless access to the Internet via node A. Node A will served as router or gateway for all nodes in this ad hoc network. In real environment of communication, ad hoc network is basically a communication among user's mobile networks. So user's devices will supportively functioning as a network that can offer network infrastructure such as routers, switches and servers. As long as within transmission range, nodes can communicate each other.

MANET originally proposed for military application, then began extend to another applications in another field. Began deployment in disaster scenario is most challenging but it can be one of significant application [2]. After disaster occurs, information of victims involved is necessary so that victims can be rescued fast. Example, Earthquake and Tsunami that happen at Great East Japan, Wenchuan at China, Tsunami at Indonesia and floods in Malaysia (e.g. Fig. 1 \& 2) extremely change the landscape. It is common results of collapse structure after earthquake. It is impossible for disaster victims make use of communication devices such as cellular phones, iPad or laptop to find others families and friends who are trapped in the disaster [3]. The devices become unworkable due to communication services that have been damaged in the area. With decentralize and infrastructure less feature, MANET can be one of the options to solve communication in disaster problem [3]. Hence, the significant functionalities of mobile ad hoc network which allows users to build dynamically reconfigurable wireless networks without fixed infrastructure was very useful [4]. 
Communication being critical after disaster happened. According to [2], the classification of disaster area can be divided into three categories; 1. Dense obstacle region (example: dense forest @ skyscrapers to urban area due to earthquake) 2. Sparse obstacle region (example: highly populated urban area) 3. Obstacles free region (playground @ stadium).

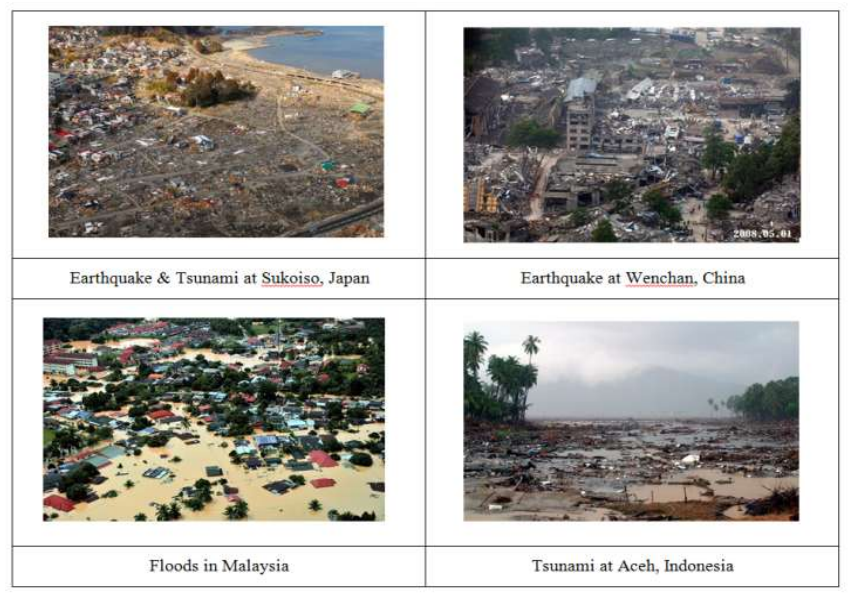

Fig. 1 Example of disaster area

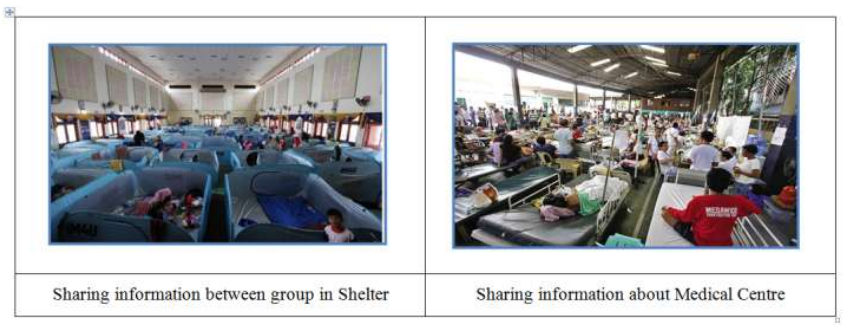

Fig. 2 Example of needed sharing information among victims

In the deployment of MANET for disaster area scenario, region can be affected by obstacles and give the problem of performance evaluation.

The main contributions of this paper are as follows:

-Introducing the enhancement of gateway load balancing scheme. This has the ability to decrease congestion in performance network.

-Presenting routing selection scheme to simplify selection of route. This has the benefit to reduce packet delay.

-Proposing gateway load balancing and routing selecting scheme to enhance network performance. Measurement is made by packet throughput and fairness.

The remainder of the paper is organized as follows: In Section 2 the state of gateway and routing in MANET are presented. In Section 3 the scenario and problem formulation are defined. Section 4 is devoted to the picture of proposed scheme while in Section 5 the simulation is described in detail. In Section 6 the discussion of simulation results and finally in Section 7 , concluding remarks and future works are drawn.

\section{RELATED WORK}

MANET nodes only can communicate with others nodes in the same networks. To communicate with nodes in the others network, example Internet, Satellite or even another MANET networks, it need the door to allow entry and exit packet from one network. In others words, we call this door as a gateway.

Gateway is also a node in MANET networks. The main task of gateway is to control network traffic between two or more different network. Router have similar function with gateway but router looks at the IP address in packet header to decide which way of packet need to go, either internal or external network. In one network, it can have several gateways. Each gateway has an average queue size to monitor. If queue size exceed pre-set threshold, congestion will occur.

To understand how the congestion happens, is like we have a game at Stadium on Saturday, all vehicles go to the same destination at the same time. Of course it will cause congestion on the road due to too many car, car are moving slowly, and at last, probably a few car will turn back. So it is the same situation with network environment. Congestion will occur when many packets want to go to the same gateway at the same time and this will drive to packet loss happens. To avoid packet loss during transmission packet from source to destination via MANET gateway, load balancing scheme is one of the solutions.

\section{A. Gateway Load Balancing Scheme}

Load balancing scheme purposely to make the task equal between all gateways. The equalization will evade from one gateway have heavy load until congest meanwhile the others gateways have a light load. Previous works [5] [6] [7] [8] [9] [10] [11] have proposed several techniques to reduce congestion in term of load balancing such as Queue Base Load Balancing Algorithm, Aggregate Interface Queue Length, Hybrid Registration Mechanism and etc. All of these techniques actually are referring and enhanced from AODV routing protocol method.

Gateway load balancing plays an important role as the way to avoid congestion. If there is more than one gateway in one network, load balancing between the gateways must be taken into account to provide QoS guarantee. ([7] proposed intelligent selection gateway scheme to allow identifying overloaded of acceptance gateway message. Although this paper did research about the integration of two networks combining MANET and Satellite, but gateway selection scheme solution are introduced only for MANET network. To continuous deliver a Quality of Services (QoS) and Quality of Experience (QoE), the MONET has approach the concept of a hybrid MANET-Satellite network as one of the solution to highly mobile, dynamic and remote environment [12].

Gateway selection process is required for communication nodes between infrastructure networks and non-infrastructure networks such as Internet and MANET. The shortest path selection is based on minimum hop count. This path will be relaying traffic between MANET to internet. Various gateway selection scheme studies to improve only single network performance such as network throughput, end-to-end delay and packet delivery ratio. [13] have proposed gateway selection scheme that improved overall network performance as well as path availability computation accuracy, path load capacity and latency. 
Case studies in pastoral area of Tibet have been used to support mobile communication efficiently. To allow people interconnect through internet, [14] proposed wireless networking architecture to connects MANET to Cellular network via Terrestrial gateway and then Cellular network will connect between the MANET and Internet. [15] used almost the same case study as [14], involve Communication Architecture for Maritime Sectors which is using the integration of Cellular, Satellite, WiMax and Wi-Fi. While access to Cellular coverage is limited, [16] introduced combination of MANET and cellular to achieve enhancement of delivery packet ratio in mobile ad hoc network.

[17] have proposed, to reach at the gateway of network, the path is choosing according to a trusted nodes and uncongested route. Thus, Manoharan \& Mohanalakshmie introduced a trust based hybrid gateway selection scheme with security elements parameters to find node trust, route trust and residual route load capacity. [18] also made the same integration research but focusing on gateway load balancing strategy. The idea was taken from AODV, one of wireless ad-hoc routing protocols and was modified to propose a new gateway selection strategy in order to distribute packet evenly between gateways in the network

\section{B. Routing in MANET}

Routing is a challenging problem in mobile wireless networks. The objective of routing is to determine the best path for the packet travel. A standard algorithm measuring for routing parameters is distance, bandwidth, delay and load for a path [1]. Routing consists of a routing protocol and routing algorithm. The task of routing protocol is to exchange the information of topology and link weights. While routing algorithm is to calculate the distance between the nodes. The standard algorithms used to compute the shortest path is Dijkstra's and Belman-Ford's [19]. This paper will focus on routing algorithm. [19] focus on analyse the performances of ant routing in mobile ad hoc network. Simulations are done in two case studies. First on static network and the second one is on dynamic network topologies. Simulation is to compare the performances of ant routing with AODV and DSR. Ant routing algorithm performs well in static network topologies but on the contrary in dynamic topologies due to limited capacity and restrictions on the buffer size. While [2] proposed an approach of dynamic selection path method which is depending on node density and obstacle. He also concern about the possibility destination selection based on number of nodes that select the same destination and node distance.

Nowadays, a number of routing protocols has been developed by researcher to find the best alternative solutions in routing. Ad Hoc routing protocol can be divided into three categories, first is proactive which it is based on routing table. It's also known as table-driven. Table-driven has the information of routing that will keep updated by nodes, no regard to when and how often such routes are wanted. Therefore, it will just simply refer to the routing table every time node wants to send a packet to specific destination. Second category is reactive. Reactive is routing on demand which means the information of route from sender to receiver will only be provided when requested. Reactive routing have achieved main goal of routing to reduce the network traffic overhead. Both categories are in flat routing. A third category is Hybrid. Hybrid routing is a combination of the advantages reactive and proactive protocols. In addition, this routing protocol identifying a zone to minimize a number of packet flooding in mobile ad hoc network when it is broadcast [20].

Generally routing protocols in MANET emphasize the shortest-path route from sender to receiver as the best solution for success ratio in network but forgot to give the guarantees of QoS. The goal of QoS routing protocol is to determine one or more paths from source to destination with the bandwidth requirement less than the total bandwidth available. Connections require sufficient bandwidth for transmission. Bandwidth is one of the critical issues especially in real time applications [21]. Real time application is as video conferences applications, Voice over Internet Protocol (VoIP), online gaming, some e-commerce transaction and etc. All of these applications require the functions to be implemented over a period of time. Respond time must less than maximum time given (usually measured in seconds). Therefore, [21] proposed a multi-path QoS multicast routing (MQMR) protocol in MANET. This protocol is to meet the needs of QoS and bandwidth requirements while sending data for real time application.

\section{PROBLEM FORMULATION}

During normal situation, most devices rely on communication infrastructure. However, after disaster occurred, it does bring the isolated network devices due to collapsed and damaged infrastructure of communication (e.g. Fig. 3). For that reason, using MANET, nodes still can communicate each other. An example, if destination node is not in the source range, then neighbour node will be as a relay to forward the message until it reach to the destination (e.g. Fig. 4 \& 5).
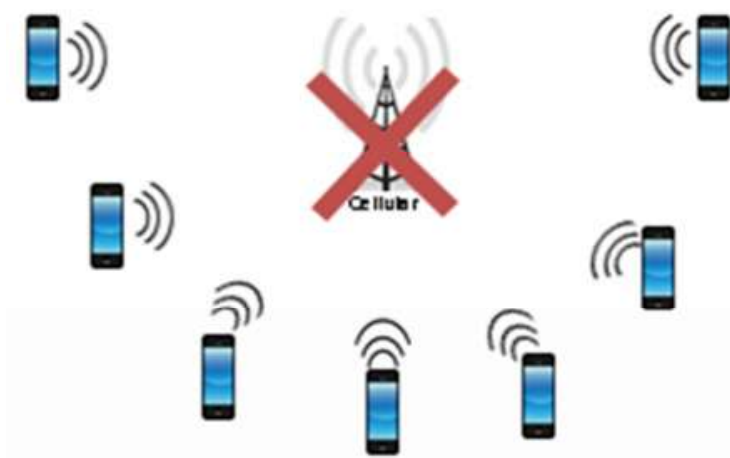

Fig. 3 Communication infrastructure collapse or damage

Nevertheless, each node within MANET network only can communicate to the nodes in the same network. While the victims attempt to connect with the Internet to let their family and friends outside know that they are safe, they want to send a message, to share the information and to share a video. However the message must go through to the internet. Therefore, to extend the communication, we proposed an enhancement of gateway load balancing and routing selection scheme and then the scheme were applied in disaster scenario. We assuming the scenario of post-disaster with electricity 
source not damage or having a backup, because in this study we are focusing on gateway load balancing and routing selection protocol to achieve better network performance. In this scenario, we assume three of MANET nodes are in Internet range. Meanwhile, the others are not. Therefore, this three nodes will be a gateway for the others node to connect with Internet.

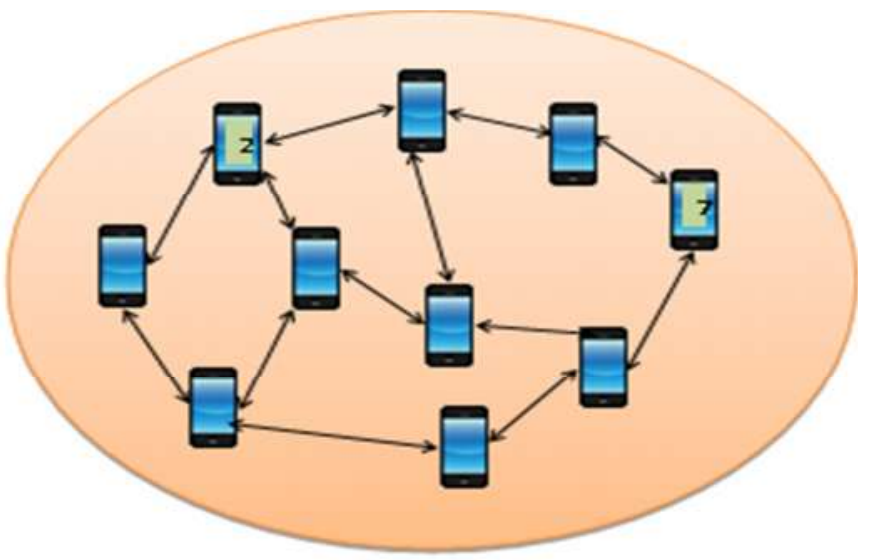

Fig. 4 MANET in disaster area

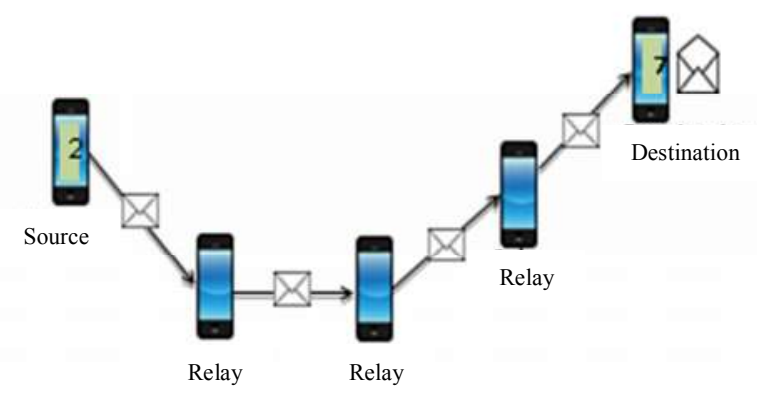

Fig. 5 Neighbour node as a relay

\section{PROPOSED SHEME}

In this study, we will compare our result with AODV routing protocol. AODV is one of routing protocol used in Mobile Ad Hoc network [22]. The scheme used hop count to find shortest path from sender to receiver. It is a good metric, simple and effective. It used reactive routing protocol. The route from sender to receiver is only established when it's needed. Sender node will broadcast RREQ (Route Request) for connection and intermediate node will forward the message until it arrived to the destination node. The broadcast technique will bring broadcast storm due to inefficiently flood the network with send messages to all nodes within range to find the best route [23]. Each node that receives the message will record temporary routes back and then routes with less hop number are chosen. Therefore, routing overhead can be reduced. However the main problem is delay because of waiting the routes connection establish first from sender to receiver. In addition, when the nodes in the network are high speed mobile it's can bring a problem as well [24].
Base on this study, we found most of the methods are modifying from AODV routing protocol. AODV use hop count as path selection but AODV not deal with load traffic. Therefore, we proposed a scheme of gateway load balancing and routing selection protocol to balance the load at gateway node and to intelligently choose the route. Our scheme used both reactive and proactive routing protocol. Before communication nodes in the network begin, gateway in MANET network will advertise their location periodically to all nodes within the range. Each node that receives the advertisement will store the information of nearest gateway in routing table. When node outside the gateway range want to send a messages, others node will forward the message until it arrive at one of the gateway. However, if gateway is in heavy load condition, the notification will be advertised. Hence, nearest node will find other nearest gateway that available. As in our previous work [25], we will use forward and backward technique to prevent from packet loss. We knew packet delay will increase as the technique used, so we made an improvements by using the notification of heavy load at the first stage.

\section{A. Proposed Gateway Load Balancing in Flowchart}

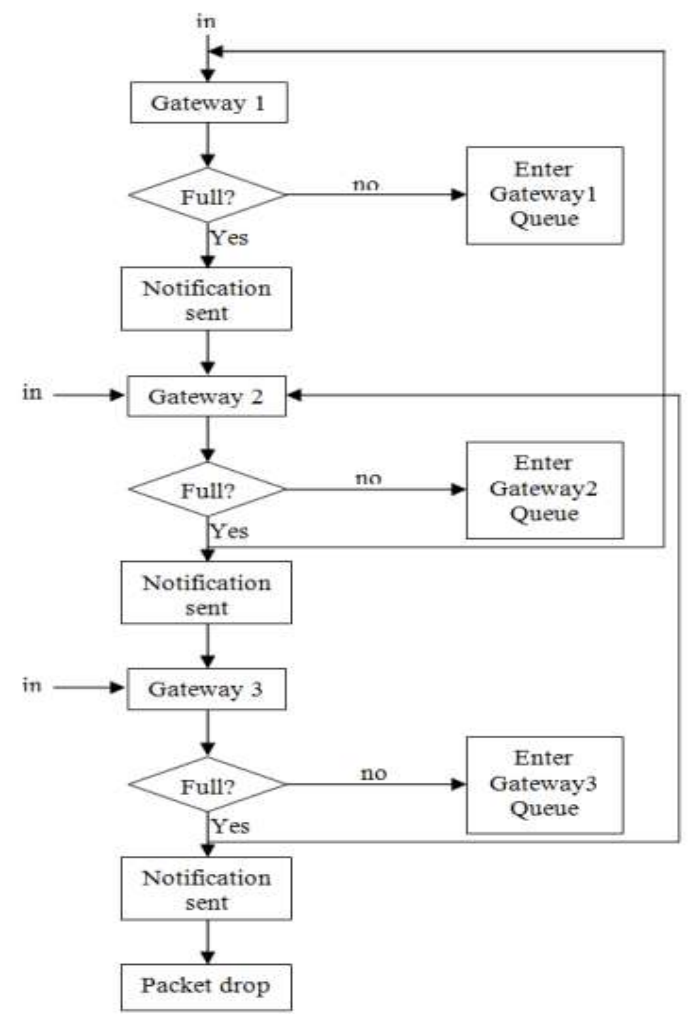

In our scenario (e.g. Fig. 6), we assume all nodes in MANET networks are isolated except three nodes that are in Internet range. Thus, the other nodes that want to communicate with nodes outside the MANET network must go through these three nodes. These nodes will be a gateway for the MANET network. Only gateway broadcast advertisement to the nearest node in their coverage range. Node that received the advertisement will keep the information of all gateways. Therefore, when the nodes 
receive a heavy notification from one of gateways, the message will not be send to that gateway. On the other hand, the message will be forwarded to other gateways that available. Each gateway has an average queue size to monitor. If queue size approaching full line, a heavy notification will be send to all nodes in coverage ranges.

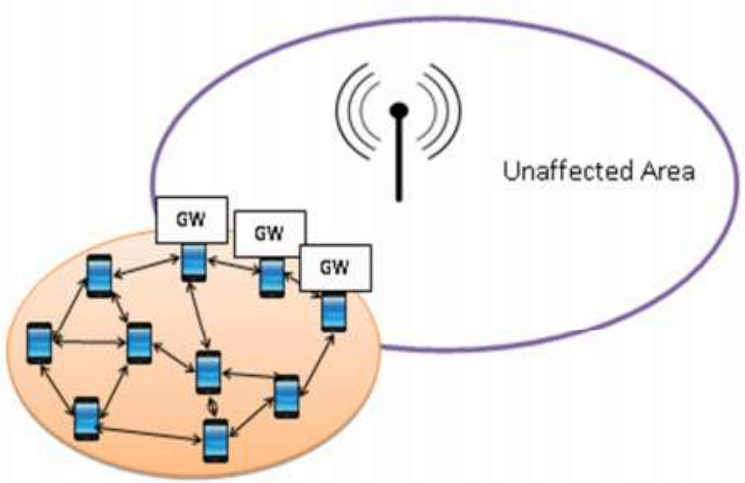

Fig. 6 MANET nodes as a Gateway

Referring to the previous work [25], our scheme will intelligently manage the transmission of message from node to gateway. To initialize the route from sender to receiver, those nodes will refer to routing table to choose which routes are available. We proposed routing selection scheme to simplify the selection of route. The following is an algorithm and flow chart of the proposed scheme:

\section{B. Proposed Routing Selection Scheme in Flowchart}

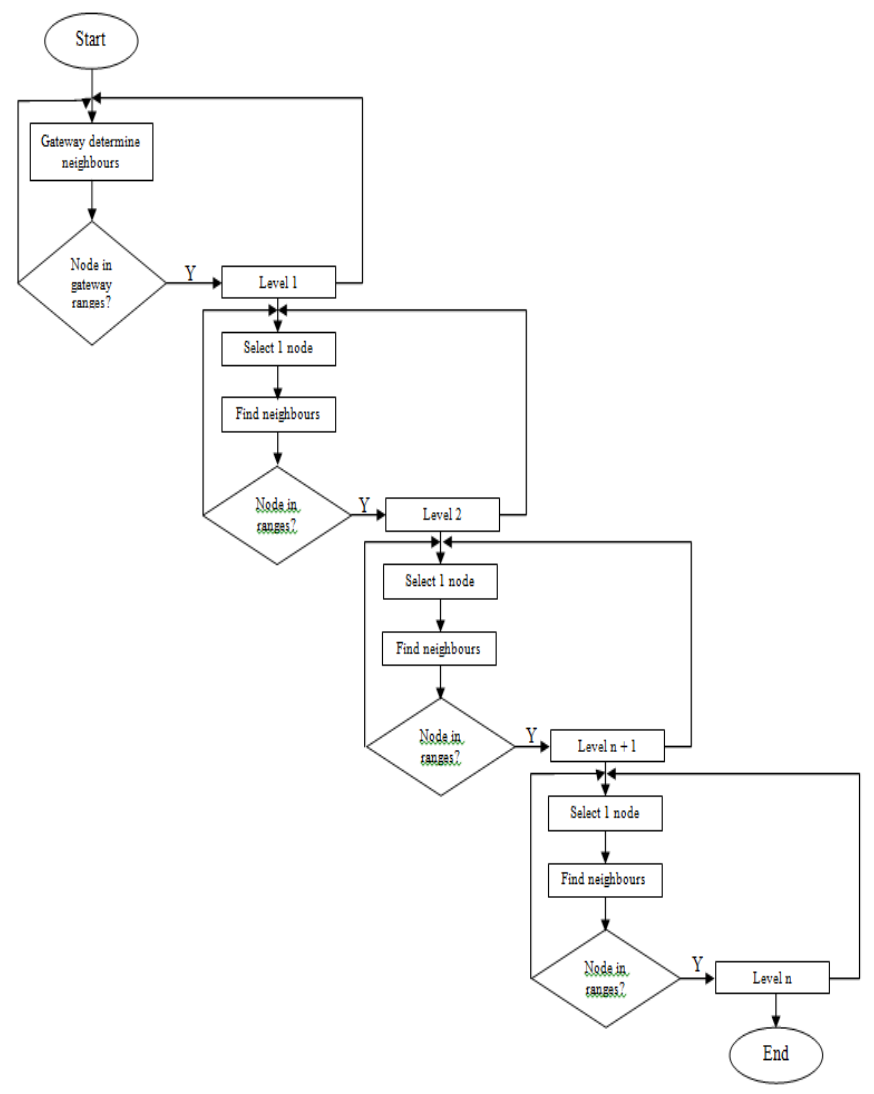

\section{Proposed Routing Selection Scheme in Algorithm}

1) Determine gateway neighbour (node within gateway coverage range)

2) All gateway neighbours listed into level 1 routing table.

3) Each node in level 1 determine their neighbours

4) Each neighbour's node of level 1 listed into level 2 routing table.

5) Redundancy node with node at level 1 will remove

6) Each node in level 2 determine their neighbours

7) Each neighbour's node of level 2 listed into level 3 routing table.

8) Redundancy node with node in level 2 will remove

9) Process repeat until all node have listed into level and no duplication of the same node at any level.

\section{SIMULATION}

The simulation of gateway load balancing scheme and gateway selection path scheme has been conducted using network simulator OMNET++. We have set MANET nodes to 50 and distributed within the area of $1200 \mathrm{~m} \times 800 \mathrm{~m}$. The transmission range of each node is equal to $250 \mathrm{~m}$. Using Random Waypoint model, the mobility speeds are set to $2 \mathrm{mps}$ and data rate is $2 \mathrm{Mbps}$. Simulation time is 900 (s).

In this simulation, we have identified host [15, 49 and 8] as a node that receive Internet coverage. Therefore, this node will be a gateway to all MANET nodes that do not have Internet coverage (e.g. Fig. 7). The destination of all messages is the gateway. Starting with gateways initialized current positions and then determine who the neighbour is, follow by node at each level determine their neighbour to find the easiest route to the gateway. Each message is passing up to higher level in sender transmission range.

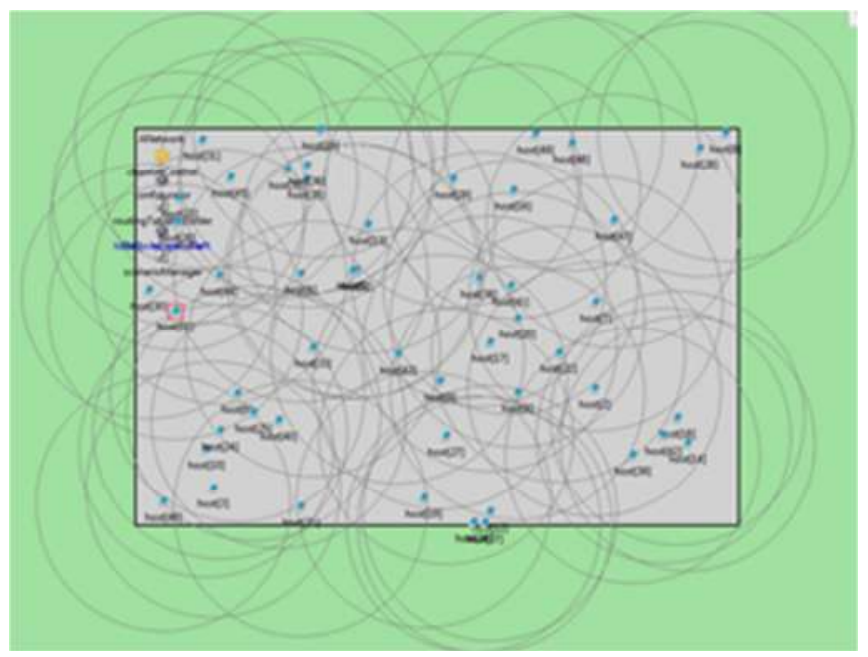

Fig. 7 Proposed schemes in simulated area. 


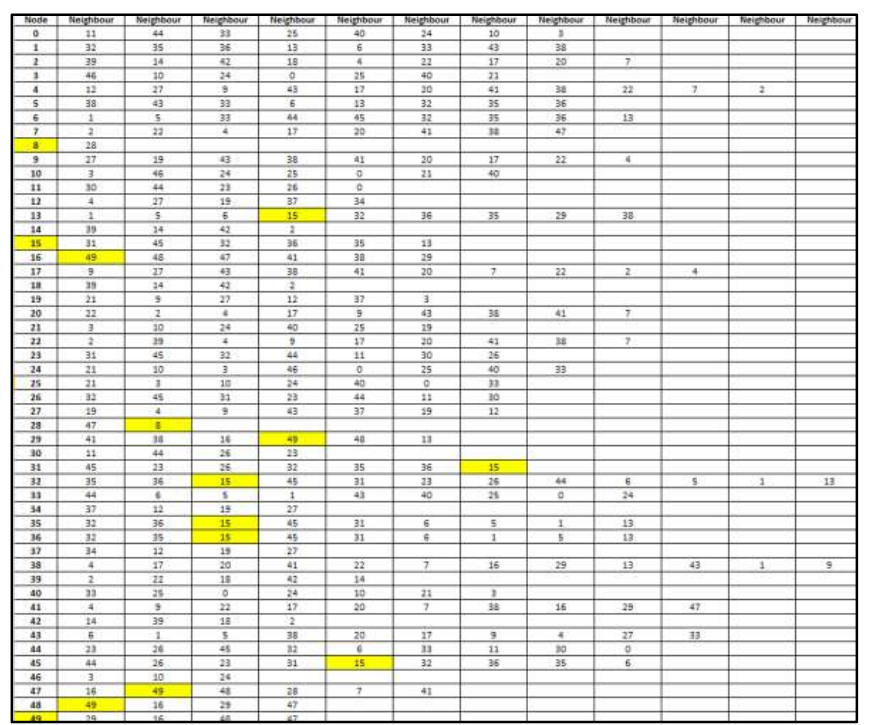

Fig. 8 The complexity of routing table.

When message received at higher level nodes and that node is not the destination, the nodes will take the responsibility to forward the message to the higher level in range. This process will be repeated until message arrives at the destination node. If higher level node is small $(\mathrm{TTL}=<1)$, message cannot be forwarded. To reduce congestion and the complexity of routing selection, we simplify routing table into several levels. Each node in that level that receives the message will forward the message into next upper level instead of broadcast to all nodes. Broadcast technique will result packet flooding in the network especially when there are many node in the networks. After applying the algorithm, the complexity of routing table (e.g. Fig. 8) can be simplified as shown below:

\begin{tabular}{|c|c|c|c|c|c|c|c|c|c|c|c|}
\hline \multirow{3}{*}{ ᄉ } & & & & & 15 & 49 & 8 & & & & \\
\hline & & & & & & & & & & & \\
\hline & 31 & 45 & 32 & 36 & 35 & 13 & 29 & 16 & 48 & 47 & 28 \\
\hline 6 & 23 & 26 & 44 & 5 & 1 & 38 & 41 & 49 & 7 & 8 & \\
\hline 33 & 11 & 30 & 0 & 43 & 4 & 17 & 20 & 22 & 9 & 2 & \\
\hline 40 & 25 & 24 & 10 & 3 & 27 & 12 & 39 & 19 & 14 & 42 & 18 \\
\hline 21 & 46 & 34 & 37 & & & & & & & & \\
\hline
\end{tabular}

Fig. 9 Routing table after the algorithm is applied.

TABLE I

FLOW OF PROPOSED ROUTING SELECTION SCHEME

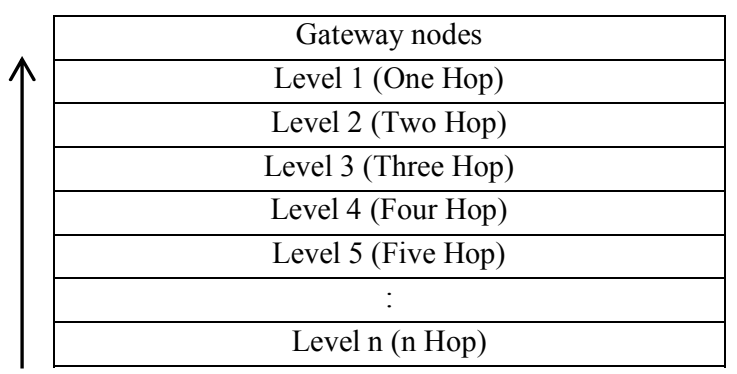

Assuming host [11] want to send a message. Host [11] is not in Internet range therefore the message must go through either host $[15,49$ or 8$]$ to get to the Internet. By using proposed routing selection scheme, host [11] is in level 3. Thus, this node will look up at the upper level which is level 2 to determine which node in level 2 is in host [11] ranges. In this case, host [44] is neighbouring to host [11]. Therefore message forwarded to host [44]. The same step repeated until the message arrives at gateway node. Total number of hop is 3 .

As shown in Fig. 9, the results of the routing table obtained after applying our proposed algorithm. The complexity of route can be simplified by using our proposed routing selection scheme. Hence, this result indicates that the total number of hop can be minimized following by shortest path determined. Interestingly, this method can be executed on any node of a gateway in Internet of Things.

\section{CONCLUSIONS}

In this paper we have proposed gateway load balancing and routing selection schemes. These schemes can be used at gateways to equal the task between all gateways and simplify the routing selection process to reduce the complexity of original routing table. Results obtained have shown that the proposed method can reduce network congestion and consequently minimize packet delays and to achieved better network throughput fairness. The performance evaluation of the proposed gateway load balancing method with node mobility is suggested as future work.

\section{REFERENCES}

B. Nancharaiah and B. Chandra Mohan, "The performance of a hybrid routing intelligent algorithm in a mobile ad hoc network," Comput. Electr. Eng., vol. 40, no. 4, pp. 1255-1264, May 2014.

J. Ebenezer, "A Mobility Model for MANET in large Scale Disaster Scenarios," in A Mobility Model for MANET in Large Scale Disaster Scenarios, 2014, pp. 59-64.

H. Nishiyama, M. Ito, and N. Kato, "Relay-by-smartphone: Realizing multihop device-to-device communications," IEEE Commun. Mag., vol. 52, no. 4, pp. 56-65, 2014.

D. G. Reina, J. M. León-Coca, S. L. Toral, E. Asimakopoulou, F. Barrero, P. Norrington, and N. Bessis, "Multi-objective performance optimization of a probabilistic similarity/dissimilarity-based broadcasting scheme for mobile ad hoc networks in disaster response scenarios," Soft Comput., vol. 18, no. 9, pp. 1745-1756, 2014.

K. Khan, "A Review of Gateway Load Balancing Strategies in Integrated Internet-MANET," in IEEE International Conference on Internet Multimedia Services Architecture and Applications(IMSAA), 2009.

[6] Q. Le-trung, P. E. Engelstad, T. Skeie, and A. Taherkordi, "LoadBalance of Intra / Inter-MANET Traffic over Multiple Internet Gateways," in MoMM '08 Proceedings of the 6th International Conference on Advances in Mobile Computing and Multimedia, 2008, no. 0316, pp. 50-57.

[7] Y. Miao, Z. Sun, F. Yao, N. Wang, and H. S. Cruickshank, "Study on Research Challenges and Optimization for Internetworking of Hybrid MANET," in Personal Satellite Services, Springer, 2013, pp. $90-101$.

[8] D. Nandiraju, L. Santhanam, N. Nandiraju, and D. P. Agrawal, "Achieving Load Balancing in Wireless Mesh Networks Through Multiple Gateways," IEEE, 2006.

[9] A. Rani and M. Dave, "Performance Evaluation of Modified AODV for Load Balancing Amita Rani and 2 Mayank Dave Department of 
Computer Science and Engineering, UIET, Department of Computer Engineering, National Institute of Technology," $J$. Comput. Sci., vol. 3, no. 11, pp. 863-868, 2007.

[10] Y. Tashtoush, O. Darwish, and M. Hayajneh, "Fibonacci sequence based multipath load balancing approach for mobile ad hoc networks," Ad Hoc Networks, vol. 16, pp. 237-246, 2014.

[11] J. H. Zhao, X. Z. Yang, and H. W. Liu, "Load-balancing Strategy of Multi-gateway for Ad hoc Internet Connectivity," in Proceeding of the International Conferences on Information Technology: Coding and Computing (ITCC'05), 2005, pp. 1-5.

[12] A. Oliveira, P. Z. Sun, M. Monier, and P. Boutry, "On Optimizing Hybrid Ad-hoc and Satellite Networks - the MONET Approach," in Future Network \& MobileSummit 2010, 2010, pp. 1-8.

[13] S. H. Bouk, I. Sasase, S. H. Ahmed, and N. Javaid, "Gateway discovery algorithm based on multiple QoS path parameters between mobile node and gateway node," J. Commun. Networks, vol. 14, no. 4, pp. 434-442, Aug. 2012.

[14] C. Huang, X. Guo, and Z. Liu, "A wireless networking architecture using MANET for mobile communications of remote pastoral areas in Tibet," in 2nd International Conference on Computer Science and Electronics Engineering (ICCSEE 2013), 2013, no. Iccsee, pp. 800-803.

[15] L. Mu, R. Kumar, and A. Prinz, "An Integrated Wireless Communication Architecture for Maritime Sector," LNCS, Springer, pp. 193-205, 2011.

[16] B. Bhargava, X. Wu, Y. I. Lu, and W. Wang, "Integrating Heterogeneous Wireless Technologies: A Cellular Aided Mobile Ad Hoc Network ( CAMA )," in Mobile Networks and Applications, Kluwer Academic Publishers, 2004, pp. 393-408.

[17] R. Manoharan and S. Mohanalakshmie, "A Trust Based Gateway Selection Scheme for Integration of MANET with Internet," in IEEE-International Conference on Recent Trends in Information Technology, ICRTIT, 2011, pp. 543-548.

[18] R.-U. Zaman, K.-U.-R. Khan, and a. V. Reddy, "A review of gateway load balancing strategies in Integrated Internet-MANET," 2009 IEEE Int. Conf. Internet Multimed. Serv. Archit. Appl., pp. 16, Dec. 2009.

[19] S. S. Dhillon, X. Arbona, and P. Van Mieghem, "Ant Routing in Mobile Ad Hoc Networks," Proceeding Third Int. Conf. Netw. Serv. (ICNS'07), Athens, Greece, no. Jun 19-25, 2007.

[20] A. K. Gupta, H. Sadawarti, and A. K. Verma, "Review of Various Routing Protocols for MANETs," Int. J. Inf. Electron. Eng., vol. 1, no. 3, 2011.

[21] N.-C. Wang and C.-Y. Lee, "A multi-path QoS multicast routing protocol with slot assignment for mobile ad hoc networks," Inf. Sci. (Ny)., vol. 208, pp. 1-13, Nov. 2012.

[22] A. Boukerche, B. Turgut, N. Aydin, M. Z. Ahmad, L. Bölöni, and D. Turgut, "Routing protocols in ad hoc networks: A survey," Computer Networks, vol. 55, no. 13. pp. 3032-3080, 2011.

[23] Y. C. Tseng, S. Y. Ni, Y. S. Chen, and J. P. Sheu, "The broadcast storm problem in a mobile ad hoc network," Wirel. Networks, vol. 8, no. 2-3, pp. 153-167, 2002.

[24] T. T. Son, H. Le Minh, G. Sexton, and N. Aslam, "A novel encounter-based metric for mobile ad-hoc networks routing," $\mathrm{Ad}$ Hoc Networks, vol. 14, pp. 2-14, 2014.

[25] N. A. Mahiddin, A. Nazari, B. Mohd, and S. Subramaniam, "An Intelligent Buffer Management For Packet Scheduling Algorithm in Multihop Wireless LANs," Int. J. Futur. Gener. Commun. Netw., vol. 5, no. 2, pp. 97-106, 2012. 